\title{
Article
}

\section{Ethical issues in dysphagia management}

\author{
Leslie, Paula and Lisiecka, Dominika
}

Available at http://clok.uclan.ac.uk/32880/

Leslie, Paula ORCID: 0000-0002-0379-9044 and Lisiecka, Dominika (2020) Ethical issues in dysphagia management. Seminars in Speech and Language, 41 (3). pp. 257-265. ISSN 0734-0478

It is advisable to refer to the publisher's version if you intend to cite from the work. http://dx.doi.org/10.1055/s-0040-1710561

For more information about UCLan's research in this area go to http://www.uclan.ac.uk/researchgroups/ and search for < name of research Group>.

For information about Research generally at UCLan please go to http://www.uclan.ac.uk/research/

All outputs in CLoK are protected by Intellectual Property Rights law, including Copyright law. Copyright, IPR and Moral Rights for the works on this site are retained by the individual authors and/or other copyright owners. Terms and conditions for use of this material are defined in the policies page.

\section{CLoK}

Central Lancashire online Knowledge www.clok.uclan.ac.uk

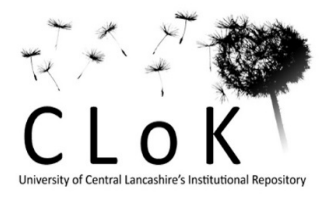




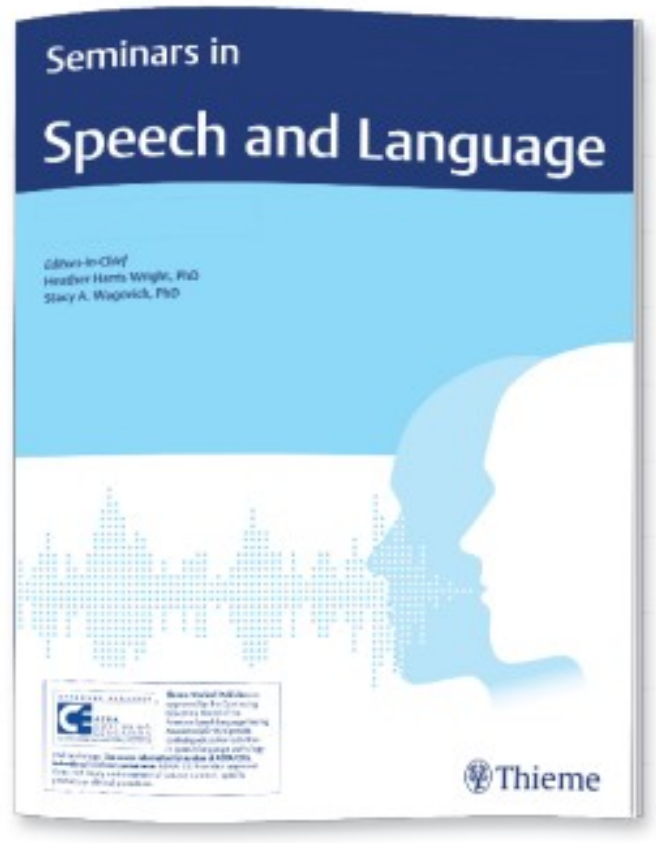

\section{Ethical issues in dysphagia management}

\begin{tabular}{|r|l|}
\hline Journal: & Seminars in Speech and Language \\
\hline Manuscript ID & SSL-20-0003-IA.R3 \\
\hline Danuscript Type: & Invited Review (Solicited) \\
\hline Author: & n/a \\
\hline Keywords: List of Authors: & $\begin{array}{l}\text { Leslie, Paula ; University of Central Lancashire, School of Sport \& Health } \\
\text { Sciences } \\
\text { Lisiecka, Dominika; Institute of Technology Tralee, Department of } \\
\text { Nursing and Healthcare Sciences }\end{array}$ \\
\hline ethics, decision making, consent, dysphagia, discretion \\
\hline & $\begin{array}{l}\text { Dysphagia management is complex and requires balancing individuals' } \\
\text { preferences, quality of life, and medical consequences. Ethical challenges } \\
\text { are not uncommon given the complexity of dysphagia. Professionals } \\
\text { must engage in ethical reflection and shared decision-making when } \\
\text { managing dysphagia. Recognizing one's own presuppositions and beliefs } \\
\text { may be fundamental to ensuring an ethical approach. } \\
\text { The goal of this article is to apply principles of ethics using hypothetical } \\
\text { case studies of dysphagia. To this end, we will describe the challenges of } \\
\text { working with the disorder of dysphagia, the influence of culture on } \\
\text { decision-making about eating and feeding, the importance of information } \\
\text { disclosure and respect for individuals' refusal of recommendations, and } \\
\text { the interplay of ethical reflection, evidence, and clinical judgment when }\end{array}$ \\
\hline
\end{tabular}


making complex dysphagia management decisions. These concepts should be kept in mind ensure compassionate and competent care of the person with eating, drinking or swallowing problems and their family caregivers.

\section{SCHOLARONE \\ Manuscripts}




\title{
Ethical issues in dysphagia management
}

\author{
Paula Leslie (corresponding author) PhD, MA Bioethics \\ Senior Lecturer Speech \& Language Therapy, FRCSLT (UK), CCC-SLP (USA) \\ School of Sport \& Health Sciences, Brook Building BB126, University of Central Lancashire, \\ Preston, PR1 2HE, United Kingdom \\ Tel: +44 (0) 1772893638 email: pleslie1@uclan.ac.uk
}

\begin{abstract}
Dominika Lisiecka PhD, MSc in Speech \& Language Therapy, MIASLT (Ireland) [1] Assistant Lecturer, Department of Nursing and Healthcare Sciences, Institute of Technology Tralee, Co. Kerry, Ireland (dominika.lisiecka@staff.ittralee.ie); [2] Post-doctoral Researcher, School of Nursing and Midwifery, University College Cork, Ireland (dominika.lisiecka@ucc.ie)
\end{abstract}




\begin{abstract}
Paula Leslie
I support clinicians and researchers from local to international level with complex decisionmaking, ethics, end of life and vulnerable populations. I am the Course Lead for the new Speech \& Language Therapy MSc being developed at the University of Central Lancashire, UK, and am interested in nontraditional routes to advanced clinical training. I serve as the Associate Coordinator for the ASHA Special Interest Group 15 (Gerontology), and on the editorial board of ASHA Special Interest Group 13 (Swallowing and Swallowing Disorders). I am a full member of the Dysphagia Research Society (USA) and a founding member and current scientific chairperson of the UK Swallow Research Group. I'm proud to be a Fellow of the Royal College of Speech and Language Therapists, to have received the SWPSHA Honors of the Association and the Louis M DiCarlo Award for contributions to palliative care and SLP work.
\end{abstract}

\title{
Dominika Lisiecka
}

I graduated with a MSc in Speech \& Language Therapy and a MSc in Pedagogy from Academy of Special Education in Warsaw, Poland in 2003. I have been working clinically in the field of dysphagia in Ireland for over 15 years. I was proud to be awarded a Research Training Fellowship for Therapy Professionals in Ireland by Health Research Board, which I completed in 2018. My $\mathrm{PhD}$ investigated the experiences of dysphagia in people with amyotrophic lateral sclerosis. In addition to clinical work I lecture in Speech \& Language Pathology, Nursing and Social Sciences. I am also a post-doctoral researcher on an international project focused on decision-making at end 


\section{Edition: Ethics through case studies}

of life in advanced dementia. I am a member of the Irish Speech \& Language Therapists association and the European Society for Swallowing Disorders. 


\begin{abstract}
Dysphagia management is complex and requires balancing individuals' preferences, quality of life, and medical consequences. Ethical challenges are not uncommon given the complexity of dysphagia. Professionals must engage in ethical reflection and shared decision-making when managing dysphagia. Recognizing one's own presuppositions and beliefs may be fundamental to ensuring an ethical approach.
\end{abstract}

The goal of this article is to apply principles of ethics using hypothetical case studies of dysphagia. To this end, we will describe the challenges of working with the disorder of dysphagia, the influence of culture on decision-making about eating and feeding, the importance of information disclosure and respect for individuals' refusal of recommendations, and the interplay of ethical reflection, evidence, and clinical judgment when making complex dysphagia management decisions. These concepts should be kept in mind ensure compassionate and competent care of the person with eating, drinking or swallowing problems and their family caregivers.

Keywords: ethics, decision-making, consent, dysphagia, discretion Financial disclosure: See COI forms from ScholarOne. 
Edition: Ethics through case studies

\section{Learner Outcomes}

The learner will be able to:

- describe the complexity of the management of dysphagia from the ethical perspective

- define the principles of autonomy, beneficence, nonmaleficence, and justice

- apply the biomedical ethical principles from the perspectives of the speech-language pathologist, the person with dysphagia and family members

- list the core tenets of evidence-based practice as applied to the management of dysphagia

\section{CEU Questions}

1) Working with people with dysphagia:

a) Raises ethically more complex issues than other areas of speech-language pathology

b) Raises ethically less complex issues than other areas of speech-language pathology

c) Raises ethical issues that are comparable to other areas of speech-language pathology practice

d) Raises ethical issues similar to those involving people with autism spectrum disorder only

e) Is ethically outside of the scope of practice for speech-language pathologists. 
Edition: Ethics through case studies

2) Autonomous decision-making by individuals with dysphagia requires professional input:

a) To support the understanding of costs and benefits of each clinical decision

b) Focused on explaining physiological processes

c) Regarding health care decisions only when individuals are at the end of life

d) Regarding health care decisions after the attending physician has made a diagnosis of the underlying condition

e) Only when mandated by a court of law

3) An expectation of all health professionals is:

a) To achieve a doctoral level education or several years practice plus Board Certification

b) To decide whether to work either in pediatric settings or adult settings

c) To identify individuals' predicaments and rights

d) To specialize in no more than three clinical areas

e) To make available at least one research study for discussion with other stakeholders

4) Cultural context (of a person with dysphagia):

a) Is not relevant when making dysphagia-specific recommendations

b) Should be established in individuals who can communicate verbally and have intact cognition

c) Is an important factor influencing the management of dysphagia

d) Is always less important than (secondary to) a medical context

e) Is an important factor influencing the decision to introduce non-oral feeding only 
Edition: Ethics through case studies

5) During the decision-making process in the management of dysphagia the SLP should:
a) Focus on medical knowledge and safety
b) Base decisions on patients' preferences only
c) Reflect on own values and cultural values and how they may impact decision-making
d) Document all key decisions in detail in patients' notes without explained them the person with dysphagia
e) Always prioritize the decisions which are cost-effective 


\section{Introduction}

Eating and drinking are vital requirements for life. How, one might ask, can there be ethical concerns with physiologic processes that are fundamental to existence? The concerns arise not from the physiologic events but rather when difficulties arise with eating, drinking, and/or swallowing. ${ }^{1}$ Dysphagia refers not merely to the biomechanics of the oropharyngeal tract but more broadly to "all aspects of swallowing, including related feeding behaviors."

Ethical issues for speech-language pathologists (SLPs) relate to the planning and delivery of services in this area. Although some clinical difficulties (and stressors) are specific to the disorder of dysphagia, the ethics principles that we use are the same across all areas of SLP practice. The purposes of this article are to describe the challenges of working with the disorder of dysphagia; the influence of culture on decision-making about eating and feeding; the importance of information disclosure and documentation; and, the interplay of ethical reflection, evidence, and clinical judgment when supporting people to make decisions regarding their complex dysphagia management.

Hypothetical cases will illustrate the resources available to SLPs to fulfill their professional responsibilities. Such resources include the knowledge and skills required by the American Speech-Language-Hearing Association (ASHA), as well as an appreciation for biomedical ethics principles, and complementary legal principles. Finally, because there is rarely a clear-cut border between right or wrong in our clinical work, learning to use both clinical judgment and ethical discretion are essential elements of competent care. 


\section{Edition: Ethics through case studies}

\section{Discomfort of Clinicians}

Reading the queries posted to online forums there is often more emotional language, more reports of sleepless nights, and more fear of losing one's license, with dysphagia than with communication issues even in similar conditions. ${ }^{1}$ Possible reasons for this are that SLPs have a longer history and a more clearly defined role in the support of people with communication issues than they do with dysphagia. Other professions have been caring for people with eating, drinking and swallowing problems as long as health care has existed and only relatively recently did the SLP move into that realm.

Learners and novice clinicians often describe a fear of working in dysphagia because "you can't kill someone with the wrong language work.” The SLP may feel under pressure or feel responsible to "sort out" some of the medical consequences of dysphagia such as repeated chest infections or weight loss, or to offer a solution when a person becomes unable to manage oral medication and a non-oral route is not appropriate. Managing progressive dysphagia may cause frustration for those with it as well as the professionals involved, as sometimes despite best efforts, a safe route for eating and drinking cannot be established.

We propose that there is a more fundamental reason for the disquiet that clinicians report. Food and drink, eating and swallowing are activities that all humans undertake across the lifespan from birth to death. Control of what, when, how, with whom usually sits with the person unless too young or unable to manage without help. 


\section{Food and Culture}

Eating and drinking are important human activities, certainly because they are vital to sustenance but also because of their importance to identity and community. Across all societies meals are usually taken with others and when restrictions are placed on what or when to eat, it tends to be for cultural reasons. ${ }^{3}$ Thus, the SLP must span two worlds: a lifetime of acculturalization to the human act and symbolism of eating and drinking, and the clinical role of limiting or modifying the act. The SLP nests their own clinical impressions and recommendations in the context of the culture within which they are most familiar. This needs to be acknowledged and addressed relative to the cultural context of the person with dysphagia.

Personal perceptions of food also come in to play with some people experiencing more pleasure from eating than others: some eat to live and some live to eat. Perceptions can change as the impact of dysphagia increases or decreases. ${ }^{4}$ This may impact how individuals engage with the SLPs' recommendations to alter food consistency or to commence non-oral feeding. How SLPs think about eating and drinking may affect their recommendations to their patients, and their reactions to patients' responses. Consider the role of certified nursing assistants (CNAs) involved in supporting people with dysphagia which runs parallel with the role of SLPs. A study of CNAs revealed two perspectives regarding feeding assistance. Social feeders viewed the mealtime as a time to engage with patients and to socialize. Technical feeders prioritized the need to ensure adequate consumption. Each group (the social feeders and the technical feeders) perceived the other as either pushy or not trying hard enough. ${ }^{5}$ Both groups knew something about swallowing problems and cared for their patients, but they approached the act of feeding differently. 
Edition: Ethics through case studies

We highlight the distinction between eating as a sociocultural event and eating as a clinicalphysiologic event. Understanding these different perspectives can illuminate why SLPs and their patients have different opinions and approaches to dysphagia management. The special nature of food (and eating and drinking) should be acknowledged in the world of clinical interventions. By reflecting on their own values and cultural mores, SLPs will deepen their appreciation for how to care effectively for individuals with dysphagia — and how to resolve ethical difficulties when they arise.

\section{Medical ethical principles}

In biomedical ethics there are several approaches or philosophies that are used to frame issues. One of these is the Principles Approach, where an ethical conundrum is considered and various principles are applied to it to help identify a solution. Other approaches include virtue ethics, feminist ethics, duty-based ethics, and case-based ethics (casuistry). ${ }^{6}$ In this article, we have adopted the four main principles outlined originally in 1979 by Beauchamp and Childress. ${ }^{7}$ For a detailed examination of ethical approaches, morality, and law, the reader is referred to Horner (2004). ${ }^{8}$ Table 1 shows the four principles and a simple definition of each.

Table 1 here 
Edition: Ethics through case studies

\section{Documentation}

The critical underpinning to evidence-based intervention, legislation, and professional standards is that the process and dialogue must be clearly documented. Such documentation must be timely, accurate, and relevant. Informed consent is not just an event or a signature on a piece of paper, it is an active process. ${ }^{9}$ In legal disputes the courts look for a clear record that relevant information was provided to a person for the decision being considered. ${ }^{10}$ Not just that information was provided but that the person sharing that information ensured that it was understood. Thus, recording statements such as "Mr. Smith was told/educated/given..." are of no use. What the professional should do is record how the person demonstrated understanding using approaches such as teach back: "Mr. Smith demonstrated understanding of the possible costs and benefits of X approach when he described how his chest is affected by his swallowing problem... he reported that the glass of beer at his weekly bridge game was important even though he might cough...". Further detail on the informed consent process ${ }^{11-13}$ and how to document informed consent is available in previous papers. ${ }^{8,14,15}$

\section{Case 1: Refusal of Modified Diet}

Case: Zak (75 years old) had a stroke five years prior and had a further stroke which his physician said was unlikely to be the last. He was now struggling to manage solid foods and coughed almost always with regular drinks. Zak refused to modify his diet despite the advice from the SLP after a clinical an instrumental examination showing aspiration on both consistencies. The SLP felt she was failing in her duty. 


\section{Edition: Ethics through case studies}

This is a classic scenario that illustrates the tug of war between the companion duties of beneficence-nonmaleficence, and the duty to respect (and develop) the autonomy of the people with whom we work.

As an autonomous individual, Zak has the right to consider health care advice and to agree to interventions, or not. Our respect for his autonomy presumes that he has been presented with the information that is relevant (material) to making the decision, that he understands the information and the consequences of his decision, and, that he can communicate his preference. The SLP has a duty to ensure understanding but not to police a decision that does not follow the SLP's preferred approach. It is important to note that the idea that autonomy (self-determination) resides with each person is a cultural (largely Western) construct. Many societies locate decisionmaking with an elder in the family group, a social leader, or among several family members. ${ }^{16}$ Understanding different cultural norms and being sensitive to individuals' perspectives is the place to start health care decision-making.

The ethical imperatives of doing good (beneficence) and preventing harm (nonmaleficence) are foundational biomedical ethical principles, rooted in the history of medicine and familiar to all health care professionals. The fundamental importance of these principles does not mean that they ethically "outweigh" the principle of respected for autonomy, or vice versa. It is necessary to consider all the principles, and particularly to weigh and balance them when actions associated the principles conflict.

As illustrated in Zak's case, interventions to prevent aspiration is the paradigm application of the nonmaleficence principle in dysphagia management. Clinicians try to avoid aspiration through diet modification, as the case of Zak illustrates. The ethical quandary arises because Zak has 
refused the diet modification and his clinician is fearful of a bad outcome. The SLP knows that it is difficult to predict how a given person will tolerate aspiration with any degree of certainty. It is also difficult to predict whether the individual can maintain adequate nutrition and hydration on the modified diet. Zak may have a good chest status with no infections, and/or he may take so long to eat that he is struggling to maintain adequate nutrition and hydration. Adherence to the principle of nonmaleficence needs to be considered through a wider lens than aspiration alone. In order to avoid harm, some SLPs are overly risk averse and recommend very restrictive diets. This then puts people directly in the path of harm from dehydration and malnutrition. ${ }^{17-19}$ SLPs may inadvertently cause harm by failing to address the complexities of the case, both clinically and ethically.

In order to adhere to the principle of justice, that is to provide what people need, requires us to explore the beliefs and preferences of our patients and families. Ultimately this reduces inappropriate or unwelcome recommendations. By addressing misunderstandings, SLPs may realize that the viewpoints of the person and the professional are much closer together and that they can agree on an alternative and more efficient intervention. Throughout an ethical deliberation, compassion and benevolence of the professional toward the patient are paramount. With these attributes at the forefront, the SLP working with an individual like Zak will listen to the person with dysphagia, bring expertise and evidence to the issues of concern, and help the patient with informed decision-making. A part of this may well be the exploration of (mis)understanding by the person with dysphagia but the SLP will not know that unless a sensitive discussion and shared decision-making take place. 


\section{Edition: Ethics through case studies}

\section{Case 2: Access to Evidence-Based Care}

The principle of justice is based on providing what people need in a fair and equitable manner (see Table 1). Application of the principle of justice, like the other medical ethical principles, is closely aligned with the evidence-based practice (EBP) framework discussed below.

Case: Nora (58 years old) was a full-time nurse when she was diagnosed with bulbar onset ALS. During the SLP assessment Nora refused to talk about ALS and her prognosis and asked the SLP to "just fix her speech and dribbling." The SLP attempted to talk about Nora's future swallowing status but Nora quickly replied that this is all not for her and to give her some exercises. The SLP demonstrated basic breathing and relaxation exercises to improve voice projection. Three weeks later Nora reported feeling ready for more active exercises. The SLP explained that the scientific evidence for exercises in saliva management in ALS is poor. Nora sighed and said: "I don't care about your evidence, just give me something to do!"

Over twenty years ago Sackett and colleagues from the centers for EBP in the UK, USA, and Canada, published a short paper titled: "Evidence-based medicine: what it is and what it isn't." 20 In their paper (for which we will replace medicine with practice) the three tenets of EBP are clearly outlined. What has happened over the years is that people focus on the first, maybe the second, and often forget the third. The cornerstone of individual preference is not something we consider after the first two tenets. Rather it is fundamental to the expertness of the clinician (see Table 2). 
Edition: Ethics through case studies

Table 2 here

True experts look carefully at the person in front of them and consider the world from that person's perspective, this can only be done with sensitive dialogue: understanding individuals' preferences helps us to support their autonomy. Then the expert brings to the table evidence and clinical wisdom to support individuals faced with making the decisions about eating and drinking. EBP as defined here does not start with randomized controlled trials (RCTs), or defensive practices to protect one's license, or following the local peer approach, or simply agreeing to whatever individuals_and their families want.

How does this help us with Nora? EBP aligns with ethical codes and professional standards in that we need to put the person with dysphagia at the center of the decision-making process. In order to increase autonomy we need to help individuals to become informed about the decisions they face: listen to their concerns, undo misunderstandings, and present balanced options. Increasing evidence is showing that people who are more engaged in their own health care have better outcomes. ${ }^{21}$ Part of the professional role is to increase the knowledge, skills, and confidence of their patients regarding their impairments. This applies equally in progressive conditions where our role is not to rehabilitate but to enable the person to manage their condition. Until we know what framework an individual person is operating from we cannot contribute the most appropriate expertise or supportive evidence. Nora's focus on her voice and dribbling might be because that is the biggest problem to her. What we are not required to do is provide treatment that is inappropriate or ineffectual. The ASHA Code of Ethics is clear on this: 
Edition: Ethics through case studies

\author{
Principle I, Rule K "Individuals who hold the Certificate of Clinical Competence \\ shall evaluate the effectiveness of services provided, technology employed, and \\ products dispensed, and they shall provide services or dispense products only \\ when benefit can reasonably be expected." 22
}

\begin{abstract}
What can be "reasonably expected" by an SLP with regard to intervention has to be based on carefully reviewed best practice. The SLP is expected to remain open to new sources of evidence across their careers and to revise their expectations accordingly.
\end{abstract}

What does "benefit" mean, and for whom, also requires consideration. For some this might be a longer life, for others a more active one. Nora may be getting psychological benefit from doing something in the face of her condition. Some may question at what point does protective denial become a problem and is onward referral necessary. When making professional recommendations to Nora it is crucial that: 1) the health care team are in effective communication with each other and the person at the center of the process, and 2) if discussions have been held along the way about the medical condition, possible costs, disadvantages and benefits of different courses of action, then Nora has been involved. This is the ideal of good planning and delivery of SLP services.

\title{
Support for Ethical Care in the Law
}

Specific legislation addressing the area of dysphagia is limited, but SLPs will find overarching principles in health care helpful. Such standards include that a person has the right to accept or refuse any treatment offered to them-if they have capacity, if adequate disclosure was given, and if consent was obtained. ${ }^{23}$ The onus is on the professional to demonstrate that these things 
happened. These patient rights are mandated in federal law, enacted in $1990 .{ }^{24}$ In turn, the Centers for Medicare and Medicaid Services have policies pertaining to informing patients of their legal rights, and of course ASHA sets out what is expected of competent clinicians in documents such as the SLP Scope of Practice and Code of Ethics. ${ }^{2,22}$

Case: Zak's SLP is now concerned that she might lose her state license or worse if Zak gets pneumonia or chokes.

Over one hundred years ago a patient brought suit against the Society of New York Hospital based on the laws at the time regarding trespass. ${ }^{25}$ The Court found that an operation performed without a patient's consent was medical battery. Justice Benjamin Cardozo summed up that in the Court's opinion:

Every human being of adult years and sound mind has a right to determine what shall be done with his own body.25

In the 21 st century, most jurisdictions in the United States treat lack of informed consent as medical negligence (rather than trespass or battery). These informed consent cases demonstrate that clinicians have a legal duty is to disclose risks and benefits of interventions, thereby supporting the right of autonomous individuals to make their own medical decisions. Zak has right to refuse medical treatment, even life-sustaining treatment like a feeding tube, if he understands the consequences adequately. This requires the responsible professional to have shared relevant information.

Although most of the formal legal cases pertain to surgeons and physicians over the years, the principles apply equally to SLPs. This is a resource for us rather than a threat. The legal duty to 
Edition: Ethics through case studies

disclose material facts means that clinicians must know how to listen to people about their concerns and must be able to explain medical conditions and interventions. Disclosure is a prerequisite for informed consent and is what the courts will look for if we are ever challenged. ${ }^{1,26}$

An important piece of federal legislation is the Patient Self-Determination Act of 1990 (PSDA), ${ }^{24}$ and the corresponding requirements laid out by the Centers for Medicare and Medicaid Services (CMS). ${ }^{27}$ The PSDA requires most Medicare and Medicaid providers (hospitals, managed care/home health organizations, nursing homes, and hospice programs) to give people specific information about their entitlements under state laws, including the right to:

- participate in and direct their own health care decisions

- $\quad$ accept or refuse medical or surgical treatment 24

The health care provider is not allowed to place conditions on the delivery of care or discriminate against someone based on whether they agree with professional recommendations. SLPs are thus required to consider the opinions, values, and wishes of the person in their care with regard to planning and undertaking treatment.

In the CMS Medicare Benefit Policy Manual, documentation of services reimbursed by Medicare must contain evidence of:

- $\quad$ skilled services provided,

- medical necessity for those services,

- the patient's response to intervention, and

- the basis for the ongoing (or discontinuation) of the plan of treatment ${ }^{27}$ 


\section{Edition: Ethics through case studies}

The person at the center of the decision-making process ("the patient") has a clear right to voice their opinion of interventions offered.

\section{Case 3: Feeding Tubes: Saints or Sinners?}

This question comes up frequently in discussions of medical conditions. The gastric feeding tube was developed in the latter part of the 20th century to aid in short term recovery after surgery for children. ${ }^{28}$ The overuse of feeding tubes in many adult populations could require a thesis of its own; for this topic, we refer the reader to other publications. ${ }^{29-36}$ Our focus is on ethical concerns about feeding tubes beyond nutrition and hydration.

Case: Juan (27 years old) presented with dysphagia related to his severe cerebral palsy. His wife used to spend hours feeding him every day. Juan recently agreed to having a feeding tube inserted after he discussed this with his clinical team. Meals taken non-orally take only a couple of minutes. Although Juan and his wife gained time, it was hard for them to engage in other joint activities due to Juan's disability. Juan's wife regretted the feeding tube as the hours spent feeding Juan in the past were precious moments of interaction and closeness with her husband.

Feeding tubes at their most simplistic allow for the biomechanical model's provision of fuel to a body. Feeding tubes were initially thought to mitigate risks associated with eating, drinking, or swallowing difficulties. We are learning that the use of feeding tubes long term can lead to an increased risk of gastric aspiration. Feeding tube regimes often take time to be established appropriately, which can cause discomfort. The general principle of non-oral feeding is to bypass the oro-pharynx and deliver nutrition and hydration in a direct, efficient way. Time spent in lengthy, distressing mealtimes impinges on family relationships. Thus, the gift of this time back 


\section{Edition: Ethics through case studies}

and reduction of stress appeals to the beneficent health care professional. Helping someone to eat and drink is a human act of caring that starts when a newborn arrives and continues until a loved one dies. ${ }^{37}$

Much more than mere fueling the body happens in the feeding transaction such as the expression of love and welcome responsibility. ${ }^{38}$ For example, spouses of people with cancer reported the pending loss of a loved one, and a double bereavement of the loss of the feeder role. ${ }^{39}$ Juan had capacity to make the decision to accept a feeding tube but what was not discussed was how this might impact his wife's experience of the feeding act. Juan's wife did not anticipate that the presence of feeding tube would reduce the time spent in meaningful activity with her husband. This would form part of the discussion with Juan and his wife, and an examination of other activities, or perhaps some pleasure tastes might form part of a solution. The SLP should be prepared to raise these issues as patients and families may not know to ask about them.

\section{Conclusion}

Evidence-based practice and patient's preferences are at the core of SLP services and this is not any different in the case of dysphagia. SLPs often feel like they face complex ethical dilemmas when dealing with a person with dysphagia. It is crucial for SLPs to integrate ethical principles to clinical work so to guide and support decisions made by the person with dysphagia, which requires that adequate time is given to establishing the management plan. SLPs should be open to addressing ethical issues from various perspectives and work with the wider team to identify the optimal solution. Much of the failing in our work and what we perceive as ethical challenges are due to poor communication rather than a maleficent professional. ${ }^{40}$ SLPs are duty bound to ensure appropriate documentation including how consent was obtained and person's 


\section{Edition: Ethics through case studies}

understanding demonstrated. It may be worthwhile to review dysphagia assessment forms/templates to ensure adequate space is provided for documenting the process of consent and clinical decision-making. To practice ethically, professionals must be competent, listen to the concerns of the person at the center of care, share relevant information, and actively engage in interprofessional team work. 
Edition: Ethics through case studies

\section{References}

1. Askren A, Leslie P. Complexity of clinical decision making: consent, capacity, and ethics. Semin Speech Lang. 2019;40(3):162-169.

2. American Speech-Language-Hearing Association. Scope of Practice in Speech-Language Pathology. ASHA. https://www.asha.org/policy/SP2016-00343/. Published 2016. Accessed December 29, 2019.

3. Counihan C, Van Esterik P, eds. Food and Culture A Reader, 3rd ed. New York, NY: Routledge; 2013.

4. Lisiecka D, Kelly H, Jackson J. How do people with Motor Neurone Disease experience dysphagia? A qualitative investigation of personal experiences. Disabil Rehabil. 2019:110. Online ahead of print. doi:10.1080/09638288.2019.1630487

5. Pelletier CA. Feeding beliefs of certified nurse assistants in the nursing home: a factor influencing practice. J Gerontol Nurs. 2005;31(7):5-10.

6. Singer PA, Viens AM. Textbook of Bioethics. Cambridge, UK: Cambridge University Press; 2008.

7. Beauchamp TL, Childress JF. Principles of Biomedical Ethics. 8th ed. New York: Oxford University Press; 2019.

8. Horner J. Morality, ethics, and law: introductory concepts. Semin Speech Lang. $2003 ; 24(4): 263-274$.

9. Meisel A, Roth LH, Lidz CW. Toward a model of the legal doctrine of informed consent. Am J Psychiatry. 1977;134(3):285-289.

10. Canterbury v. Spence, 464 F.2d 772, 150 U.S. App. D.C. 263 (1972). 
11. Sharp HM. Informed Consent in Clinical and Research Settings: What Do Patients and Families Need to Make Informed Decisions? SIG 13 Perspect Swallowing Swallowing Disorders (Dysphagia). 2015;24(4):130-139.

12. American Medical Association. Informed Consent. AMA Website.. Published 2020. Accessed 1 March, 2020.

13. Siegal G, Bonnie R, Appelbaum P. Personalized disclosure by information-on-demand: attending to patients' needs in the informed consent process. J Law Med Ethics. 2012;40(2):359-367.

14. American Speech-Language-Hearing Association. Documentation in Health Care. ASHA. Practice Portal: Professional Issues Web site. http://www.asha.org/PracticePortal/Professional-Issues/Documentation-in-Health-Care/. Published 2019. Accessed 31 December, 2019.

15. Sharp HM, Bryant KN. Ethical issues in dysphagia: when patients refuse assessment or treatment. Semin Speech Lang. 2003;24(4):285-299.

16. Kleinman A. The Illness Narratives. New York: Basic Books; 1988.

17. Rattray M, Desbrow B, Roberts S. Comparing nutritional requirements, provision and intakes among patients prescribed therapeutic diets in hospital: an observational study. Nutrition. 2017;39-40:50-56.

18. Hedman S, Nydahl M, Faxen-Irving G. Individually prescribed diet is fundamental to optimize nutritional treatment in geriatric patients. Clin Nutr. 2015;e-pub.

19. Wright L, Cotter D, Hickson M. The effectiveness of targeted feeding assistance to improve the nutritional intake of elderly dysphagic patients in hospital. J Hum Nutr Diet. 2008;21(6):555-562; quiz 564-555. 
Edition: Ethics through case studies

20. Sackett DL, Rosenberg WM, Gray JA, Haynes RB, Richardson WS. Evidence based medicine: what it is and what it isn't. BMJ. 1996;312(7023):71-72.

21. Hibbard JH, Greene J. What the evidence shows about patient activation: better health outcomes and care experiences; fewer data on costs. Health Affairs. 2013;32(2):207-214.

22. American Speech-Language-Hearing Association. Code of Ethics. ASHA. http://www.asha.org/Code-of-Ethics/. Published 2016. Updated 1 March, 2016. Accessed 29 December, 2019.

23. Appelbaum PS, Grisso T. Assessing patients' capacities to consent to treatment. New Eng J Med. 1988;319(25):1635-1638.

24. Patient Self-Deterrmination Act of 1990. Omnibus Budget Reconciliation Act of 1990, P. L. 101-508, 42 U.S.C. 1395 cc(f) (Medicare), 1396a(w) (Medicaid).

Patient Self Determination Act of $1990, \S 1395 \mathrm{cc}$ (a) (1).

25. Schloendorff v. Society of New York Hospital, 92, 211 NY 125 (1914).

26. Leslie $\mathrm{P}, \mathrm{Crawford} \mathrm{H}$. The concise guide to decision making and ethics in dysphagia. Guildford, UK: J\&R Press Ltd; 2017.

27. Centers for Medicare \& Medicaid Services. Medicare Benefit Policy Manual 100-02. In. Vol 15. Baltimore, MD: Department of Health \& Human Services; 2014.

28. Minard G. The history of surgically placed feeding tubes. Nutr Clin Pract. 2006;21(6):626-633.

29. Royal College of Physicians and British Society of Gastroenterology. Oral Feeding Difficulties and Dilemmas: A Guide to Practical Care, Particularly Towards the End of Life. London: Royal College of Physicians; Feb 2010. 1470-2118. 
30. Gomes GF, Pisani JC, Macedo ED, Campos AC. The nasogastric feeding tube as a risk factor for aspiration and aspiration pneumonia. Curr Opin Clin Nutr Metab Care. 2003;6(3):327-333.

31. Fromme E. Should my loved one get a feeding tube? Palliat Med. 2004;7(5):735.

32. Iizuka M, Reding M. Use of percutaneous endoscopic gastrostomy feeding tubes and functional recovery in stroke rehabilitation: a case-matched controlled study. Arch Phys Med Rehabil. 2005;86(5):1049-1052.

33. Walker A. In the absence of food. Food; Cult Soc. 2005;8(2):161-180.

34. Dunlop JT. The Feeding Tube Dilemma. The Center for Bioethics \& Human Dignity, Deerfield, IL: Trinity International University. https://cbhd.org/content/feeding-tubedilemma-key-questions. Published 2006. Accessed 26 July, 2014.

35. Teno J, Gozalo P, Mitchell S, et al. Does feeding tube insertion and its timing improve survival? J Am Geriatr Soc. 2012;60(10):1918-1921.

36. Regnard C, Leslie P, Crawford H, Matthews D, Gibson L. Gastrostomies in dementia: bad practice or bad evidence? Age Ageing. 2010;39(3):282-284.

37. Miller L, Rozin P, Fiske AP. Food sharing and feeding another person suggest intimacy; two studies of American college students. Eur J Soc Psychol. 1998;28(3):423-436.

38. Bergstresser SM, Castellanos E. Feeding versus artificial nutrition and hydration: at the boundaries of medical intervention and social interaction. Int $J$ Feminist Approaches Bioeth. 2015;8(2):204-225.

39. Bell K, Lee J, Ristovski-Slijepcevic S. Perceptions of food and eating among Chinese patients with cancer: findings of an ethnographic study. Cancer Nurs. 2009;32(2):118126. 
Edition: Ethics through case studies

40. Leslie P, Casper M. Ethical challenges: less about moral wrongdoing and more about communication breakdown. SIG 15 Perspect Gerontol. 2015;20(3):72-84. 
Edition: Ethics through case studies

Table 1 Biomedical Ethical Principles 7

\begin{tabular}{|l|l|}
\hline Principle & Description \\
\hline Autonomy & Respect another's worth and right to make choices \\
\hline Beneficence & Take positive action to do good for others and to prevent or remove harm \\
\hline Nonmaleficence & Deliberately avoid causing harm \\
\hline Justice & Provide what people need in a fair and equitable manner \\
\hline
\end{tabular}


Edition: Ethics through case studies

Table 2 Cornerstones of EBP as described by Sacket et al (1996) 20

\begin{tabular}{|l|l|}
\hline EBP component & Sacket et al quote \\
\hline Evidence & $\begin{array}{l}\text { Evidence based medicine is the conscientious, explicit, and judicious use of } \\
\text { current best evidence in making decisions about the care of individual } \\
\text { patients }\end{array}$ \\
\hline $\begin{array}{l}\text { Clinician } \\
\text { expertise }\end{array}$ & $\begin{array}{l}\text { clinical expertise with the best available external clinical evidence from } \\
\text { systematic research. By individual clinical expertise we mean the proficiency } \\
\text { and judgment that individual clinicians acquire through clinical experience } \\
\text { and clinical practice. }\end{array}$ \\
\hline Patient preference & $\begin{array}{l}\text { Increased expertise is reflected in many ways, but especially in more } \\
\text { effective and efficient diagnosis and in the more thoughtful identification and } \\
\text { compassionate use of individual patients' predicaments, rights, and } \\
\text { preferences in making clinical decisions about their care }\end{array}$ \\
\hline
\end{tabular}

